Homology, Homotopy and Applications, vol.4(1), 2002, pp.87-101

\title{
MODULE DERIVATIONS AND NON TRIVIALITY OF AN EVALUATION FIBRATION
}

\author{
KATSUHIKO KURIBAYASHI \\ (communicated by Charles A. Weibel)
}

\begin{abstract}
We give a sufficient condition for the evaluation fibration, whose total space is the free iterated loop space, not to be totally non cohomologous to zero with respect to a given field.
\end{abstract}

\section{Introduction}

Let $\mathbb{F}_{p}$ the prime field with $p$ elements if $p \neq 0$ and let $\mathbb{F}_{0}$ denote the rational number field $\mathbb{Q}$. We say that a fibration $F \stackrel{i}{\longrightarrow} E \stackrel{p}{\longrightarrow} B$ is totally non cohomologous to zero (henceforth TNCZ) with respect to the field $\mathbb{F}_{p}$ if the induced map $i^{*}: H^{*}\left(E ; \mathbb{F}_{p}\right) \rightarrow H^{*}\left(E ; \mathbb{F}_{p}\right)$ is surjective, equivalently, the Leray-Serre spectral sequence for the fibration collapses at the $E_{2}$-term.

Let $X$ be an $n$-connected space of finite type with a base point and $\Lambda^{m} X$ the $m$-fold free loop space $\operatorname{map}\left(S^{m}, X\right)$, namely, the space of all continuous maps from the $m$-dimensional sphere $S^{m}$ to $X$. For $1 \leqslant m \leqslant n$, let us consider the evaluation fibration

$$
\mathcal{F}_{m}: \Omega^{m} X \longrightarrow \Lambda^{m} X \stackrel{e v}{\longrightarrow} X,
$$

where $e v$ is the evaluation map defined by $e v(\gamma)=\gamma(0)$ for $\gamma \in \Lambda^{m} X$. One may ask when the evaluation fibration $\mathcal{F}_{m}$ is TNCZ. The purpose of this paper is to give a sufficient condition for the evaluation fibration $\mathcal{F}$ not to be TNCZ with respect to $\mathbb{F}_{p}$. To this end, we first take a note of some non-trivial relation between indecomposable elements in the cohomology algebra $H^{*}\left(X ; \mathbb{F}_{p}\right)$. Such a relation brings us a non-trivial relation in $H^{*}\left(\Lambda^{m} X ; \mathbb{F}_{p}\right)$ via the Eilenberg-Moore spectral

Acknowledgements: The author wishes to thank Sadok Kallel for his interest in this work and helpful comments on the proofs of Theorems 1.1 and 1.2.

Received May 22, 2002, revised August 16, 2002; published on November 5, 2002.

2000 Mathematics Subject Classification: 55T20, 57T35, 55S05.

Key words and phrases: Module derivation, evaluation fibration, the Eilenberg-Moore spectral sequence, Whitehead product.

(C) 2002, Katsuhiko Kuribayashi. Permission to copy for private use granted. 
sequences associated with fibre squares

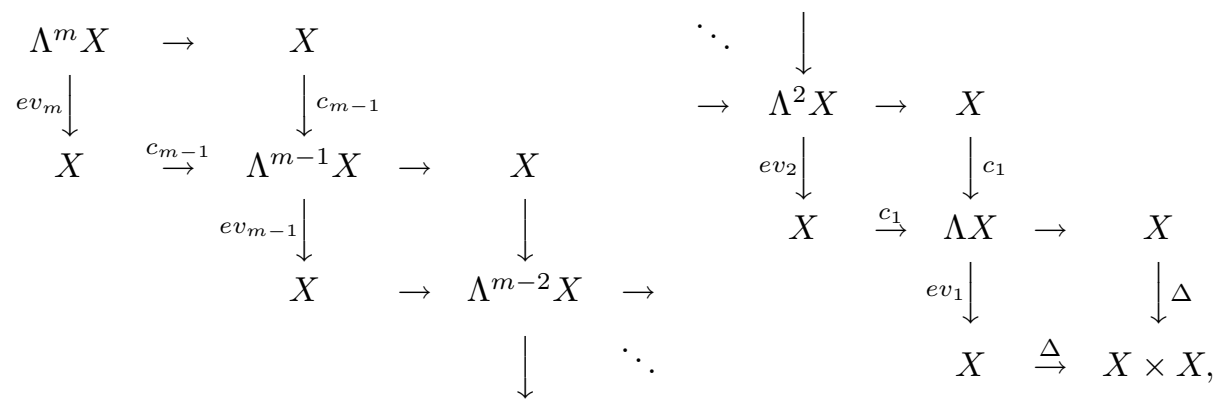

in which $\Delta$ is the diagonal map and the map $c_{i}$ is defined by carrying an element $x$ of $X$ to the constant loop at $x$. In consequence, we will see that there exists a non-trivial differential in the Leray-Serre spectral sequence. The module derivation, which has been introduced and studied in [10], plays an important role in the consideration. In fact our main theorem (Theorem 2.2) describes how to deduce the non-triviality of $\mathcal{F}_{m}$ from a non-trivial relation in $H^{*}\left(X ; \mathbb{F}_{p}\right)$ using the module derivation.

Let $F \stackrel{i}{\longrightarrow} E \stackrel{p}{\longrightarrow} B$ be a fibration with a section. Recently, Kallel and Sjerve [8] have related the brace product of the fibration, which has been introduced by James [6], to some differential in the integral homology Leray-Serre spectral sequence for the fibration. Since the evaluation fibration $\mathcal{F}_{m}$ has a section $c_{m}$ defined previously, we can detect a non-trivial differential in the spectral sequence for the fibration $\mathcal{F}_{m}$ if the brace product of the fibration is non-trivial. Observe that, in this case, the brace product is viewed as the Whitehead product up to the iterated adjoint isomorphism on the homotopy. As mentioned above, we obtain a way to deduce the non-triviality of $\mathcal{F}_{m}$ using the module derivation so that, conversely, such information on the non-triviality enables us to investigate the Whitehead products via the result due to Kallel and Sjerve.

Let $[]:, \pi_{k}(X) \otimes \pi_{l}(X) \rightarrow \pi_{k+l-1}(X)$ be the Whitehead product, $h: \pi_{s}(X) \rightarrow$ $H_{s}(X)$ the Hurewicz map and let $a d^{m}: \pi_{n}(X) \rightarrow \pi_{n-m}\left(\Omega^{m} X\right)$ denote the iterated adjoint map.

Consider the composition map

$\rho_{p} \circ h \circ a d^{m} \circ[]:, \pi_{n}(X) \otimes \pi_{n}(X) \rightarrow H_{2 n-m-1}\left(\Omega^{m} X\right) \stackrel{\rho_{p}}{\longrightarrow} H_{2 n-m-1}\left(\Omega^{m} X ; \mathbb{F}_{p}\right)$,

where $\rho_{p}$ is the $\bmod p$ reduction. Let $\xi: H^{n}\left(X ; \mathbb{F}_{p}\right) \otimes H^{n}\left(X ; \mathbb{F}_{p}\right) / \operatorname{Im}(1-T) \rightarrow$ $H^{2 n}\left(X ; \mathbb{F}_{p}\right)$ be the map induced by the cup product, in which $T$ is the homomorphism on $H^{n}\left(X ; \mathbb{F}_{p}\right) \otimes H^{n}\left(X ; \mathbb{F}_{p}\right)$ defined by $T(x \otimes y)=(-1)^{n} y \otimes x$. We can then compare the dimension of the image by the composition map $\rho_{p} \circ h \circ a d^{m} \circ[$, with that of the kernel of the map $\xi$. More precisely, we will establish the following interesting inequality.

Theorem 1.1. Let $p$ be an odd prime or zero and $X$ an $(n-1)$-connected space $(n \geqslant 2)$. Then, for $m \leqslant n-1$,

$$
\operatorname{dim} \operatorname{Im}\left(\rho_{p} \circ h \circ a d^{m} \circ[,]\right) \geqslant \operatorname{dim} \operatorname{Ker} \xi .
$$


Moreover, if $\xi \equiv 0$, then

$$
\operatorname{dim} \operatorname{Im}\left(\rho_{p} \circ h \circ a d^{m} \circ[,]\right)=\frac{1}{2} s(s+1)-\varepsilon s,
$$

where $\operatorname{dim} H_{n}\left(X ; \mathbb{F}_{p}\right)=s$ and $\varepsilon=0$ or 1 as $n$ is even or odd.

In the case $p=2$, the above (in)equality does not hold in general. Indeed, the result [8, Lemma 4.4] due to Kallel and Sjerve implies that $\rho_{p} \circ h \circ a d^{m} \circ\left[\iota_{n}, \iota_{n}\right]=0$ for the generator $\iota_{n}$ of $\pi_{n}\left(S^{n}\right)$ although $n$ is even. The Whitehead products are trivial for an $H$-space. So one can see that the condition $\xi \equiv 0$, which deduces the equality, can not be relaxed even though $p$ is odd. (We can give $\Omega S U(m)$ as such an example.) It is important to mention that Theorem 1.1 recovers an inequality concerning the Whitehead product due to Chen [2, Theorem 2]. The same argument as in the proof of Theorem 1.1 enables us to obtain an estimate of the dimension of the image by the composition $\rho_{p} \circ h \circ a d^{m} \circ[]:, \pi_{n}(X) \otimes \pi_{n+1}(X) \rightarrow H_{2 n-m}\left(\Omega^{m} X ; \mathbb{F}_{p}\right)$.

Theorem 1.2. Let $p$ be a prime number or zero and $X$ an $(n-1)$-connected space $(n \geqslant 2)$. Then, for $m \leqslant n-1$,

$\operatorname{dim} \operatorname{Im}\left\{\rho_{p} \circ h \circ a d^{m} \circ[]:, \pi_{n}(X) \otimes \pi_{n+1}(X) \rightarrow H_{2 n-m}\left(\Omega^{m} X ; \mathbb{F}_{p}\right)\right\} \geqslant \operatorname{dim} \operatorname{Ker} \zeta$, where $\zeta: H^{n}\left(X ; \mathbb{F}_{p}\right) \otimes H^{n+1}\left(X ; \mathbb{F}_{p}\right) \rightarrow H^{2 n+1}\left(X ; \mathbb{F}_{p}\right)$ denotes the cup product. $X$.

When $p=0$, we have the following theorem by analyzing the minimal model for

Theorem 1.3. (1) The inequality in Theorem 1.1 becomes an equality if $p=0$.

(2) The inequality in Theorem 1.2 becomes an equality if $p=0$ and the multiplication $m_{2}: H^{2}(X ; \mathbb{Q}) \otimes H^{2}(X ; \mathbb{Q}) \rightarrow H^{4}(X ; \mathbb{Q})$ is a monomorphism.

This paper is organized as follows. In Section 2, after recalling briefly the Koszul resolution and the module derivation, we describe our main theorem and its applications. Section 3 is devoted to proving the main theorem. In Section 4, we prove Theorems 1.1, 1.2 and 1.3.

\section{Main theorem and its applications}

In order to describe our main theorem, we need algebraic notation and terminology. For any non-negatively graded vector space $V$ of finite type over $\mathbb{F}_{p}$, we denote by $S(V)$ the symmetric algebra generated by $V$. Let $\Gamma(V)$ be the divided power algebra generated by $V$. The desuspension $s^{-1} V$ is the graded vector space defined by $\left(s^{-1} V\right)^{i}=V^{i+1}$ and we denote by $s^{-1} v \in s^{-1} V$ the element which corresponds to $v \in V$.

We here recall results on the torsion products.

Lemma 2.1. ([15, Proposition 3.5], [9, Propositions 1.1, 1.5] ) Let $A$ be a symmetric algebra $S(V)$ over $\mathbb{F}_{p}$. 
(i) There exists a projective resolution $\mathcal{K} \bullet \stackrel{\varphi}{\longrightarrow} A \rightarrow 0$ of $A$ as a left $A \otimes A$-module such that $\mathcal{K}^{\bullet}=A \otimes A \otimes \Gamma\left(s^{-1} V\right), d\left(s^{-1} u\right)=u \otimes 1-1 \otimes u$ for $u \in V$ and $\varphi$ is the multiplication of $A$, where bideg $s^{-1} u=(-1, \operatorname{deg} u)$. Hence, as a bigraded algebra,

$$
\operatorname{Tor}_{A \otimes A}(A, A) \cong S(V) \otimes \Gamma\left(s^{-1} V\right)
$$

(ii) Let $B^{\bullet}(A \otimes A, A) \rightarrow A \rightarrow 0$ be the bar resolution of $A$ as a left $A \otimes A$-module. Then there exists an isomorphism of algebras

$$
\Psi: \operatorname{Tor}_{A \otimes A}(A, A)_{b a r} \stackrel{\cong}{\longrightarrow} \operatorname{Tor}_{A \otimes A}(A, A)_{K T}
$$

such that $\Psi(1[u \otimes 1-1 \otimes u] 1)=s^{-1} u$ for $u \in V$. Here $\operatorname{Tor}_{A \otimes A}(A, A)_{b a r}$ and $\operatorname{Tor}_{A \otimes A}(A, A)_{K T}$ denote the torsion products obtained from the bar resolution and the Koszul-Tate resolution which is defined in (i), respectively.

Following [10], we define the module derivation $\mathfrak{D}: A \rightarrow \operatorname{Tor}_{A \otimes A}^{*, *}(A, A)$ by $\mathfrak{D}(a)=1[a \otimes 1-1 \otimes a] 1$ for any graded commutative algebra $A$ over $\mathbb{F}_{p}$. The map $\mathfrak{D}$ enjoys the following property:

$$
\mathfrak{D}(a b)=(-1)^{(\operatorname{deg} a+1) \operatorname{deg} b} b \mathfrak{D}(a)+(-1)^{\operatorname{deg} a} a \mathfrak{D}(b)
$$

for any $a, b \in A$. In particular, if $A$ is taken to be the symmetric algebra $S(V)$, then $\mathfrak{D}(v)=s^{-1} v$ for any $v \in V$ up to the isomorphism $\Psi$. Observe that the image of the module derivation $\mathfrak{D}$ is in $\operatorname{Tor}_{A \otimes A}^{-1, *}(A, A)$.

Let $H^{*}$ be a graded simply connected commutative algebra over $\mathbb{F}_{p}$ and $Q H^{*}$ denote the vector space of indecomposable elements. Choosing a section $s: Q H^{*} \rightarrow$ $H^{*}$ of the projection $H^{*} \rightarrow Q H^{*}=H^{*} / \bar{H}^{*} \cdot \bar{H}^{*}$, we define a surjective algebra map $q: A=S\left(Q H^{*}\right) \rightarrow H^{*}$ by $q(v)=s(v)$ for $v \in Q H^{*}$. Put $A=S\left(Q H^{*}\right)$. It follows from Lemma 2.1 that $\operatorname{Tor}_{A \otimes A}(A, A) \cong S\left(Q H^{*}\right) \otimes \Gamma\left(s^{-1} Q H^{*}\right)$ as an algebra. In particular, $\operatorname{Tor}_{A \otimes A}^{-1, *}(A, A) \cong S\left(Q H^{*}\right) \otimes s^{-1} Q H^{*}$ as an $A$-module. Therefore we can define a morphism of $A$-modules

$$
\eta: \operatorname{Tor}_{A \otimes A}^{-1, *}(A, A) \rightarrow H^{*} \otimes S\left(s^{-1} Q H^{*}\right)
$$

by $\eta\left(a \otimes s^{-1} v\right)=q(a) \otimes s^{-1} v$.

For any simply connected space $Y$, let $\sigma^{*}: H^{*}\left(Y ; \mathbb{F}_{p}\right) \rightarrow H^{*-1}\left(\Omega Y ; \mathbb{F}_{p}\right)$ denote the cohomology suspension. We are now ready to describe our main theorem.

Theorem 2.2. Let $X$ be an $n$-connected space and $m$ an integer which is less than or equal to $n$. Suppose that there exist a subspace $V \subset Q H^{*}\left(X ; \mathbb{F}_{p}\right)$ and an element $\rho \in \operatorname{Ker} q \cap S(V) \subset S\left(Q H^{*}\left(X ; \mathbb{F}_{p}\right)\right)$ such that

$$
\left.\underbrace{\sigma^{*} \circ \cdots \circ \sigma^{*}}_{m \text {-times }}\right|_{V}: V \rightarrow H^{*}\left(\Omega^{m} X ; \mathbb{F}_{p}\right)
$$

is a monomorphism and the image of $\rho$ under the composition map

$$
\eta \mathfrak{D}: A=S\left(Q H^{*}\left(X ; \mathbb{F}_{p}\right)\right) \rightarrow \operatorname{Tor}_{A \otimes A}^{-1, *}(A, A) \rightarrow H^{*}\left(X ; \mathbb{F}_{p}\right) \otimes S\left(s^{-1} Q H^{*}\left(X ; \mathbb{F}_{p}\right)\right)
$$

is non zero. Then the evaluation fibration $\Omega^{m} X \rightarrow \Lambda^{m} X \rightarrow X$ is not TNCZ with respect to $\mathbb{F}_{p}$. 
Remark 2.3. If $X$ is $n$-connected, then the cohomology suspension $\sigma^{*}: H^{i}\left(X ; \mathbb{F}_{p}\right) \rightarrow$ $H^{i-1}\left(\Omega X ; \mathbb{F}_{p}\right)$ is a monomorphism for $i \leqslant 2 n+1$. (see [17, (6.5)Corollary]). Therefore it follows that the $m$-fold cohomology suspension $\sigma^{*} \circ \cdots \circ \sigma^{*}: H^{i}\left(X ; \mathbb{F}_{p}\right) \rightarrow$ $H^{i-m}\left(\Omega^{m} X ; \mathbb{F}_{p}\right)$ is a monomorphism for $i \leqslant 2 n-m+2$. Moreover, the map $\eta \mathfrak{D}$ is computable if algebra generators in $H^{*}\left(X ; \mathbb{F}_{p}\right)$ and relations between the elements are clarified. From these facts, one can expect that the sufficient condition in Theorem 2.2 is reasonable.

In order to prove Theorem 2.2, we will rely on the Eilenberg-Moore spectral sequences which are obtained from $m$ fibre squares. The construction of the fibre square is as follows: Let us consider the fibration $\Omega^{m} X \longrightarrow X^{I^{m}} \stackrel{\text { res }}{\longrightarrow} X^{\partial I^{m}}$, where res is defined by $\operatorname{res}(\gamma)=\left.\gamma\right|_{\partial I^{m}}$. We define the map $c_{m-1}: X \rightarrow X^{\partial I^{m}}$ by $c_{m-1}(x)(t)=x$ for $x \in X$ and $t \in \partial I^{m}$. The pullback of the fibration by the map $c_{m-1}: X \rightarrow X^{\partial I^{m}}$ is regarded as the $m$-fold free loop space $\Lambda^{m} X=\operatorname{map}\left(S^{m}, X\right)$. Moreover the map res : $X^{I^{m}} \rightarrow X^{\partial I^{m}}$ can be replaced by the map $c_{m-1}: X \rightarrow$ $\Lambda^{m-1} X$ with the homotopy equivalence $\widetilde{c}: X \rightarrow X^{I^{m}}$ defined by $\widetilde{c}(x)(t)=x$ for $x \in X$ and $t \in I^{m}$. Since the space $X^{\partial I^{m}}$ can be viewed as the $(m-1)$-fold free loop space $\Lambda^{m-1} X$, we have a fibre square $\mathcal{F} S_{m}(X)$ :

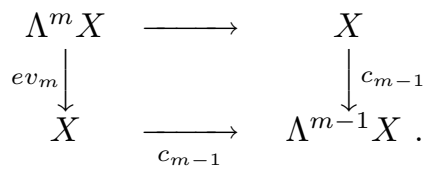

Observe that the fibration on the left hand side in $\mathcal{F} S_{m}(X)$ is the evaluation fibration $\Omega^{m} X \rightarrow \Lambda^{m} X \rightarrow X$ and that $\mathcal{F} S_{1}(X)$ is the fibre square which Smith has introduced and studied in [15]. Suppose that $X$ is an $m$-connected space. Then the fibre square $\mathcal{F} S_{m}(X)$ gives rise to the Eilenberg-Moore spectral sequence $\left\{{ }_{m} E_{r}^{*, *}, d_{r}\right\}$ converging to $H^{*}\left(\Lambda^{m} X ; \mathbb{F}_{p}\right)$ with

$$
{ }_{m} E_{2}^{*, *} \cong \operatorname{Tor}_{H^{*}\left(\Lambda^{m-1} X ; \mathbb{F}_{p}\right)}^{*, *}\left(H^{*}\left(X ; \mathbb{F}_{p}\right), H^{*}\left(X ; \mathbb{F}_{p}\right)\right)
$$

as a bigraded algebra.

An important point in proving Theorem 2.2 is what we can translate information of some relation on elements in $H^{*}\left(X ; \mathbb{F}_{p}\right)$ to that in $H^{*}\left(\Lambda^{m} X ; \mathbb{F}_{p}\right)$ via the module derivation $\mathfrak{D}: H^{*}\left(X ; \mathbb{F}_{p}\right) \rightarrow{ }_{1} E_{2}^{-1, *}$ and $H^{*}\left(X ; \mathbb{F}_{p}\right)$-module maps with degree -1 which are defined below.

Let $A$ and $M$ be simply connected graded commutative algebras and $\phi: A \rightarrow M$ an algebra map. We regard $M$ as an $A$-bimodule via $\phi$. The map $\widetilde{\mathfrak{D}}: \operatorname{Ker} \phi \rightarrow$ $\operatorname{Tor}_{A}(M, M)$ defined by $\widetilde{\mathfrak{D}}(a)=1[a] 1$ is an $A$-module map.

Lemma 2.4. $\widetilde{\mathfrak{D}}(a b)=(-1)^{\operatorname{deg} a} a[b] 1$ for $a \in A$ and $b \in \operatorname{Ker} \phi$.

Proof. Let $\partial: B^{*}(M, A, M) \rightarrow B^{*-1}(M, A, M)$ be the external differential of the bar complex which induces the torsion product $\operatorname{Tor}_{A}(M, M)$. Then, for $a \in A$ and $b \in \operatorname{Ker} \phi$, we see that $\partial(1[a \mid b] 1)=a[b] 1+(-1)^{\operatorname{deg} a+1}[a b]-(-1)^{\operatorname{deg} a+1} 1[a] b=$ $a[b] 1+(-1)^{\operatorname{deg} a+1}[a b]$. This completes the proof.

We conclude this section with examples. 
Example 2.5. Let $X$ be an $(n-1)$-connected space $(n \geqslant 1)$ and put $r_{X}=\inf \{i \geqslant$ $\left.2 \mid H^{i}\left(X ; \mathbb{F}_{p}\right) \neq 0\right\}$. Assume that $r_{\Sigma X}$ is even and $p \neq 2$ or $\operatorname{dim} H^{r_{\Sigma X}}\left(\Sigma X ; \mathbb{F}_{p}\right) \geqslant 2$. Then, for $m \leqslant n$, the evaluation fibration $\Omega^{m} \Sigma X \rightarrow \Lambda^{m} \Sigma X \rightarrow \Sigma X$ is not TNCZ with respect to $\mathbb{F}_{p}$.

Proof. Let $\left\{x_{i}\right\}$ be a basis of $V=H^{r_{\Sigma X}} H^{*}\left(\Sigma X ; \mathbb{F}_{p}\right)$. Assume that $r_{\Sigma X}$ is even and $p \neq 2$. Then, for any element $x_{i}$, we see that $x_{i}^{2} \in \operatorname{Ker} q \cap S(V)$ and $\eta \mathfrak{D}\left(x_{i}^{2}\right)=$ $2 x_{i}\left(s^{-1} x_{i}\right) \neq 0$ in $H^{*}\left(X ; \mathbb{F}_{p}\right) \otimes S\left(s^{-1} Q H^{*}\left(X ; \mathbb{F}_{p}\right)\right)$. Suppose that $\operatorname{dim} H^{r_{\Sigma X}}\left(\Sigma X ; \mathbb{F}_{p}\right) \geqslant 2$. It is readily seen that $x_{i} x_{j} \in \operatorname{Ker} q \cap S(V)(i \neq j)$ and that

$$
\eta \mathfrak{D}\left(x_{i} x_{j}\right)=(-1)^{r_{\Sigma X}}\left(x_{j} s^{-1} x_{i}\right)+(-1)^{r_{\Sigma X}}\left(x_{i} s^{-1} x_{j}\right) \neq 0
$$

in $H^{*}\left(X ; \mathbb{F}_{p}\right) \otimes S\left(s^{-1} Q H^{*}\left(X ; \mathbb{F}_{p}\right)\right)$. Since the map $\left(\sigma^{*}\right)^{(m)}: H^{r_{\Sigma X}}\left(\Sigma X ; \mathbb{F}_{p}\right) \rightarrow$ $H^{r_{\Sigma X}-m}\left(\Omega^{m} \Sigma X ; \mathbb{F}_{p}\right)$ is isomorphism, the result follows from Theorem 2.2

In the case $m=1$, we can obtain a characterization for the evaluation fibration $\Omega \Sigma X \rightarrow \Lambda \Sigma X \rightarrow \Sigma X$ to be TNCZ. Indeed, the cohomology of the free loop space $\Lambda Y$ of a simply connected space $Y$ is isomorphic to the Hochschild homology of the singular cochain complex of $Y$ as a vector space $([7])$. Moreover since suspension spaces are $\mathbb{K}$-formal in the sense of Anick (or El haouari) for any field $\mathbb{K}([\mathbf{1}])$, it follows that $H^{*}\left(\Lambda \Sigma X ; \mathbb{F}_{p}\right)$ is isomorphic to the Hochschild homology $H H\left(H^{*}\left(\Sigma X ; \mathbb{F}_{p}\right)\right)$. Therefore direct computation of the Hochschild homology of spheres leads us to the following result.

Example 2.6. Suppose that $X$ is a connected space. Then the evaluation fibration $\Omega \Sigma X \rightarrow \Lambda \Sigma X \rightarrow \Sigma X$ is $\mathrm{TNCZ}$ with respect to $\mathbb{F}_{p}$ if and only if $H^{*}\left(X ; \mathbb{F}_{p}\right) \cong$ $H^{*}\left(S^{2 k} ; \mathbb{F}_{p}\right)$ for some $k$ and $p$ is odd or $H^{*}\left(X ; \mathbb{F}_{p}\right) \cong H^{*}\left(S^{m} ; \mathbb{F}_{p}\right)$ for some $m$ and $p=2$.

One may expect that the evaluation fibration $\mathcal{F}_{m}(X): \Omega^{m} X \rightarrow \Lambda^{m} X \rightarrow X$ is TNCZ with respect to $\mathbb{F}_{p}$ if and only if the cohomology algebra $H^{*}\left(X ; \mathbb{F}_{p}\right)$ is free because the assertion is true if $p=0$. However it is not true in general when $p>0$. In fact the evaluation fibration $\mathcal{F}_{1}(\mathbb{C} P(n))$ over the complex project space is TNCZ with respect to $\mathbb{F}_{p}$ if and only if $n+1 \equiv 0 \bmod p$ (see $[\mathbf{9}$, Theorem 2]).

Since it is difficult to determine the cohomology of the $m$-fold free loop space in general, we can not deduce easily that a give evaluation fibration is TNCZ. Theorem 2.2 is applicable to the case where $X$ is the Stiefel manifold.

Example 2.7. For any $1 \leqslant m<n$, the evaluation fibration

$$
\Omega^{m} S O(n+k) / S O(n) \longrightarrow \Lambda^{m} S O(n+k) / S O(n) \longrightarrow S O(n+k) / S O(n)
$$

is not TNCZ with respect to $\mathbb{F}_{p}$ if $n$ is even and $p$ is odd.

Proof. Put $X=S O(n+k) / S O(n)$. As is known $[\mathbf{1 1}], H^{*}\left(X ; \mathbb{F}_{p}\right) \cong$

$$
\left\{\begin{array}{l}
\wedge\left(e_{2 n+3}, \ldots, e_{2 n+2 k-3}\right) \otimes \mathbb{F}_{p}\left[x_{n}\right] /\left(x_{n}^{2}\right) \text { if } n \text { is even and } k \text { is odd } \\
\wedge\left(e_{2 n+3}, \ldots, e_{2 n+2 k-5}, e_{n+m-1}^{\prime}\right) \otimes \mathbb{F}_{p}\left[x_{n}\right] /\left(x_{n}^{2}\right) \text { if } n \text { and } k \text { are even } .
\end{array}\right.
$$


We choose a 1-dimensional subvector space $V$ of $Q H^{*}\left(X ; \mathbb{F}_{p}\right)$ so that $V=\mathbb{F}_{p}\left\{x_{n}\right\}$. Since the element $x_{n}$ is of the least degree, it follows that $\left.\sigma^{*} \circ \cdots \sigma^{*}\right|_{V}$ is a monomorphism (see Remark 2.3). Moreover we see that $\eta \mathfrak{D}\left(x_{n}^{2}\right)=2 x_{n} s^{-1} x_{n} \neq 0$ in $H^{*}\left(x ; \mathbb{F}_{p}\right) \otimes S\left(s^{-1} Q H^{*}\left(X ; \mathbb{F}_{p}\right)\right)$. By virtue of Theorem 2.2 , we have the result.

\section{Proof of the main theorem}

For based spaces $Y$ and $X$, let $\operatorname{map}_{*}(Y, X)$ denote the space of all based maps from $Y$ to $X$. Throughout this section, we assume that $X$ is an $n$-connected space. For any integer $m \leqslant n$, let us consider a morphism of fibre squares

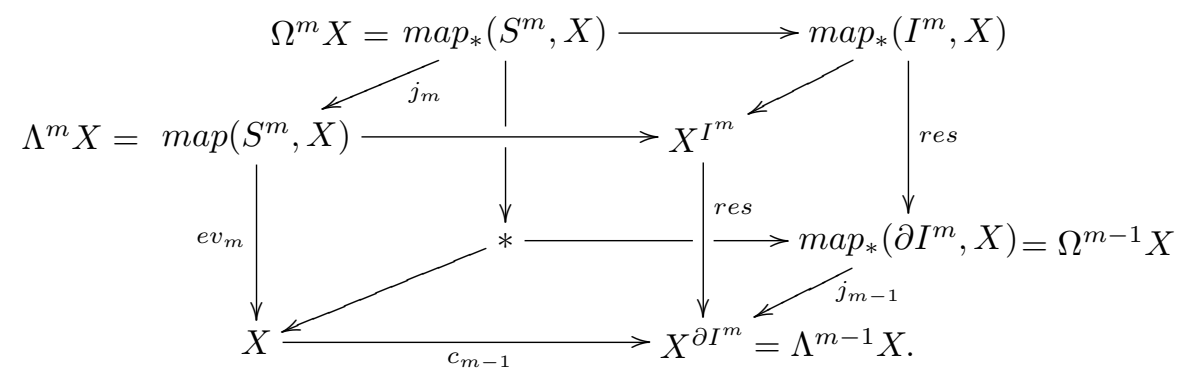

Observe that the back square gives rise to the Eilenberg-Moore spectral sequence $\left\{{ }_{m} \hat{E}_{r}^{*, *}, \hat{d}_{r}\right\}$ converging to $H^{*}\left(\Omega^{m} X ; \mathbb{F}_{p}\right)$ with

$$
{ }_{m} \hat{E}_{2}^{*, *} \cong \operatorname{Tor}_{H^{*}\left(\Omega^{m-1} X ; \mathbb{F}_{p}\right)}^{* *}\left(\mathbb{F}_{p}, \mathbb{F}_{p}\right)
$$

as a bigraded algebra. For the rest of this section, the cohomology algebra $H^{*}\left(X ; \mathbb{F}_{p}\right)$ will be denoted by $H^{*}$ and the coefficient fields of the cohomologies will be omitted when no confusion results. Let $\left\{{ }_{m} f_{r}\right\}:\left\{{ }_{m} E_{r}^{*, *}, d_{r}\right\} \rightarrow\left\{{ }_{m} \hat{E}_{r}^{*, *}, \hat{d}_{r}\right\}$ be the morphism of spectral sequences induced from the above morphism of fibre squares. Let $\left\{{ }_{m} F^{-i}\right\}_{i \geqslant 0}$ and $\left\{{ }_{m} \hat{F}^{-j}\right\}_{j \geqslant 0}$ denote the filtrations of the Eilenberg-Moore spectral sequences $\left\{{ }_{m} E_{r}^{*, *}, d_{r}\right\}$ and $\left\{{ }_{m} \hat{E}_{r}^{*, *}, \hat{d}_{r}\right\}$, respectively. The naturality of the morphism of spectral sequences allows us to obtain the following lemma.

Lemma 3.1. The following two diagrams consist of commutative squares:

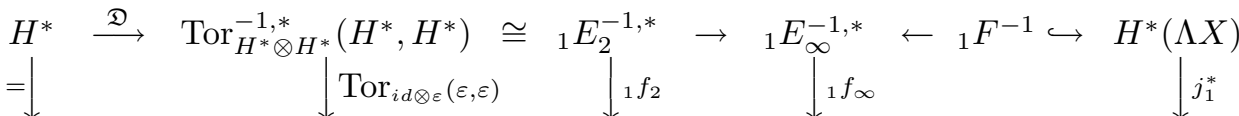

$$
\begin{aligned}
& H^{*} \stackrel{\widetilde{\mathfrak{D}}_{\Omega}}{\longrightarrow} \operatorname{Tor}_{H^{*}}^{-1, *}\left(\mathbb{F}_{p}, \mathbb{F}_{p}\right) \cong{ }_{1} \hat{E}_{2}^{-1, *} \rightarrow{ }_{1} \hat{E}_{\infty}^{-1, *}={ }_{1} \hat{F}^{-1} \hookrightarrow H^{*}(\Omega X), \\
& \operatorname{Ker} c_{m-1}^{*} \quad \stackrel{\widetilde{\mathfrak{D}}}{\longrightarrow} \operatorname{Tor}_{H^{*}\left(\Lambda^{m-1} X\right)}^{-1, *}\left(H^{*}, H^{*}\right) \cong{ }_{m} E_{2}^{-1, *} \rightarrow \cdots \\
& j_{m-1}^{*} \downarrow \downarrow \operatorname{Tor}_{j_{m-1}^{*}}(\varepsilon, \varepsilon) \_\downarrow_{m} f_{2} \\
& H^{*}\left(\Omega^{m-1} X\right) \stackrel{\widetilde{\mathcal{D}}_{\Omega}}{\longrightarrow} \operatorname{Tor}_{H^{*}\left(\Omega^{m-1} X\right)}^{-1, *}\left(\mathbb{F}_{p}, \mathbb{F}_{p}\right) \cong{ }_{m} \hat{E}_{2}^{-1, *} \rightarrow \cdots
\end{aligned}
$$

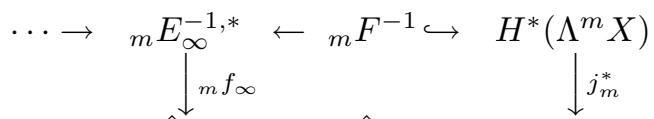

$$
\begin{aligned}
& \cdots \rightarrow{ }_{m} \hat{E}_{\infty}^{-1, *}={ }_{m} \hat{F}^{-1} \hookrightarrow H^{*}\left(\Omega^{m} X\right) .
\end{aligned}
$$


Here $\widetilde{\mathfrak{D}}_{\Omega}$ is the map defined by mapping $x$ to $[x], \mathfrak{D}$ and $\widetilde{\mathfrak{D}}$ are the module derivation and the $H^{*}$-module map defined in $\S 2$, respectively, and $\varepsilon: H^{*} \rightarrow \mathbb{F}_{p}$ is the augmentation.

The upper sequences in Lemma 3.1 are covered by more algebraic sequences.

Lemma 3.2. (i) There exist an $H^{*}$-module map $\widetilde{\alpha}_{1}: H^{*} \otimes s^{-1} Q H^{*} \rightarrow{ }_{1} F^{-1}=$ $F^{-1} H^{*}\left(\Lambda X ; \mathbb{F}_{p}\right)$ and an $H^{*}$-algebra map $\alpha_{1}: H^{*} \otimes S\left(s^{-1} Q H^{*}\right) \rightarrow H^{*}\left(\Lambda X ; \mathbb{F}_{p}\right)$ such that the following two diagrams are commutative:

$$
\begin{aligned}
& \begin{array}{ccc}
A=S\left(Q H^{*}\right) & \stackrel{\mathcal{D}}{\longrightarrow} & \operatorname{Tor}_{A \otimes A}^{-1, *}(A, A) \\
q \downarrow & & \\
H^{*} & \stackrel{\mathcal{D}}{\longrightarrow} & \operatorname{Tor}_{H^{*} \otimes H^{*}}^{-1, *}\left(H^{*}, H^{*}\right)
\end{array} \\
& \stackrel{(I)}{\cong} S\left(Q H^{*}\right) \otimes s^{-1} Q H^{*} \stackrel{q \otimes 1}{\longrightarrow} H^{*} \otimes s^{-1} Q H^{*} \quad \hookrightarrow \quad H^{*} \otimes S\left(s^{-1} Q H^{*}\right) \\
& \cong{ }_{1} E_{2}^{-1, *} \rightarrow{ }_{1} E_{\infty}^{-1, *} \longleftarrow \quad \longleftarrow \quad{ }_{1} F^{-1} \quad \hookrightarrow \quad H^{*}(\Lambda X), \\
& \begin{array}{ccc}
A_{1}:=H^{*} \otimes S\left(s^{-1} Q H^{*}\right) & \stackrel{\phi_{1}}{\longrightarrow} & H^{*} \\
\left.\alpha_{1}\right\rfloor & & \rfloor= \\
H^{*}(\Lambda X) & \stackrel{c_{1}^{*}}{\longrightarrow} & H^{*},
\end{array}
\end{aligned}
$$

where $\phi_{1}: H^{*} \otimes S\left(s^{-1} Q H^{*}\right) \rightarrow H^{*}$ is defined by $\phi_{1}\left(h \otimes s^{-1} v\right)=0$ and $\phi_{1}(h \otimes 1)=h$. (ii) There exist an $H^{*}$-module map $\widetilde{\alpha}_{m}: H^{*} \otimes\left(s^{-1}\right)^{(m)} Q H^{*} \rightarrow F^{-1} H^{*}\left(\Lambda^{m} X ; \mathbb{F}_{p}\right)$ $={ }_{m} F^{-1}$ and an $H^{*}$-algebra map $\alpha_{m}: H^{*} \otimes S\left(\left(s^{-1}\right)^{(m)} Q H^{*}\right) \rightarrow H^{*}\left(\Lambda^{m} X ; \mathbb{F}_{p}\right)$ such that the following two diagrams are commutative:

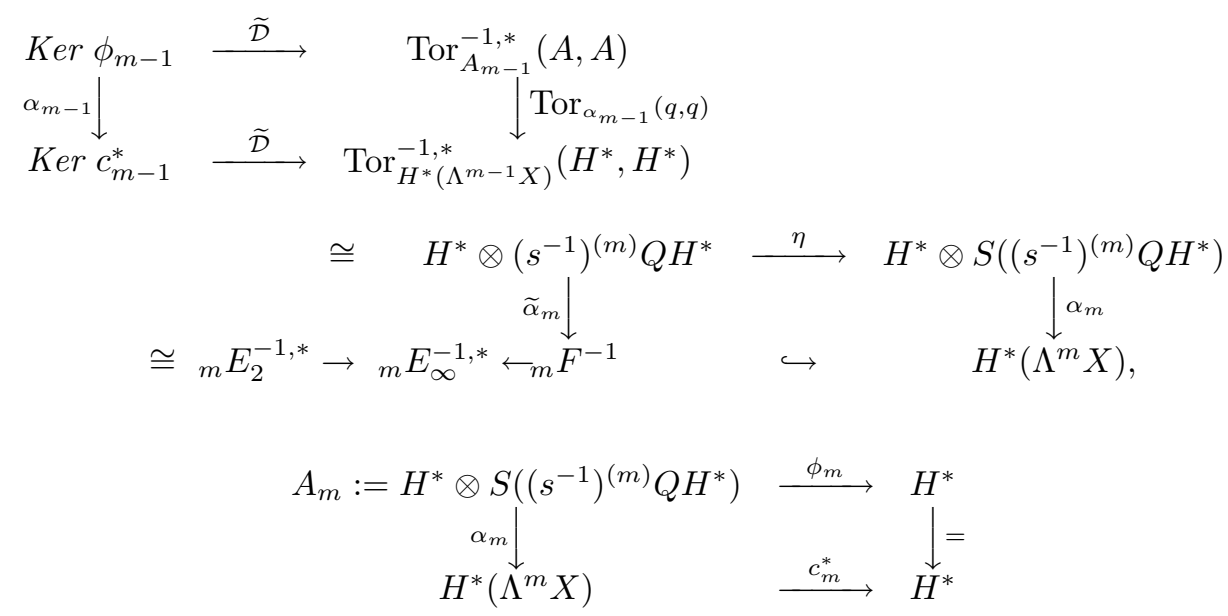

where $\left(s^{-1}\right)^{(m)} Q H^{*}$ denotes the $m$-fold desuspension of the vector space $Q H^{*}$ and 
$\phi_{m}: H^{*} \otimes S\left(\left(s^{-1}\right)^{(m)} Q H^{*}\right) \rightarrow H^{*}$ is defined by $\phi_{m}\left(h \otimes\left(s^{-1}\right)^{(m)} v\right)=0$ and $\phi_{m}(h \otimes$ 1) $=h$.

By virtue of Lemma 2.1, we can obtain the isomorphism (I). Observe that the composition map $S\left(Q H^{*}\right) \otimes s^{-1} Q H^{*} \stackrel{q \otimes 1}{\longrightarrow} H^{*} \otimes s^{-1} Q H^{*} \hookrightarrow H^{*} \otimes S\left(s^{-1} Q H^{*}\right)=A_{1}$ coincides with the map $\eta$ defined before Theorem 2.2 .

Proof of Lemma 3.2. We define $H^{*}$-module map $\widetilde{\alpha}_{1}: H^{*} \otimes s^{-1} Q H^{*} \rightarrow F^{-1} H^{*}(\Lambda X)$ by $\widetilde{\alpha}_{1}\left(s^{-1} v\right)=1[q(v) \otimes 1-1 \otimes q(v)] 1$, where $1[q(v) \otimes 1-1 \otimes q(v)] 1$ denotes a representative element of $\{\mathfrak{D}(q(v))\}$ such that $c_{1}^{*}(1[q(v) \otimes 1-1 \otimes q(v)] 1)=0$. Moreover, choosing $1\left[\widetilde{\alpha}_{m-1}\left(s^{-1}\right)^{(m-1)} v\right] 1$ as a representative element of $\left\{\widetilde{\mathfrak{D}}\left(\left(s^{-1}\right)^{(m-1)} v\right)\right\} \in$ ${ }_{m} E_{\infty}^{-1, *}$, we define $H^{*}$-module maps $\widetilde{\alpha}_{m}: H^{*} \otimes\left(s^{-1}\right)^{(m)} Q H^{*} \rightarrow F^{-1} H^{*}\left(\Lambda^{m} X\right)$ by $\widetilde{\alpha}_{m}\left(\left(s^{-1}\right)^{(m)} v\right)=1\left[\widetilde{\alpha}_{m-1}\left(s^{-1}\right)^{(m-1)} v\right] 1$ inductively. Since the evaluation fibration $e v_{m}: \Lambda^{m} X \rightarrow X$ has a section $c_{m}: X \rightarrow \Lambda^{m} X$, there is no loss in generality in supposing that $c_{m}^{*}\left(1\left[\widetilde{\alpha}_{m-1}\left(\left(s^{-1}\right)^{(m-1)} v\right)\right] 1\right)=0$. We have the required maps $\widetilde{\alpha}_{1}$ and $\widetilde{\alpha}_{m}$.

The module map $\widetilde{\mathfrak{D}}$ defined in $\operatorname{Ker} \phi_{m-1}$ or Ker $c_{m-1}^{*}$ will be denoted below by $\widetilde{\mathfrak{D}}_{m}$. From the definitions of $\mathfrak{D}$ and $\widetilde{\mathfrak{D}}_{m}$, we see that $\operatorname{Im} \eta \mathfrak{D} \subset \operatorname{Ker} \phi_{1}$ and that $\operatorname{Im} \eta \widetilde{\mathfrak{D}}_{i-1} \subset \operatorname{Ker} \phi_{i}$. This fact allows us to compose the maps $\mathfrak{D}, \widetilde{\mathfrak{D}}_{m}$ and $\eta$ 's as $\eta \widetilde{\mathfrak{D}}_{m-1} \cdots \eta \widetilde{\mathfrak{D}}_{1} \eta \mathfrak{D}$.

Lemma 3.3. The following diagram is commutative:

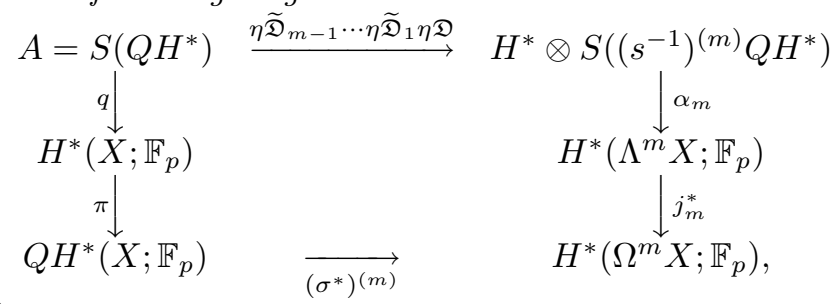

where $\left(\sigma^{*}\right)^{(m)}$ is the m-fold suspension map.

Proof. It follows from [13, Proposition 4.5] that the composition map $H^{*}\left(\Omega^{i-1} X\right) \stackrel{\mathfrak{D}_{\Omega}}{\longrightarrow} \operatorname{Tor}_{H^{*}\left(\Omega^{i-1} X\right)}^{-1, *}\left(\mathbb{F}_{p}, \mathbb{F}_{p}\right) \cong{ }_{i} \hat{E}_{2}^{-1, *} \rightarrow{ }_{i} \hat{E}_{\infty}^{-1, *}=F^{-1} \rightarrow H^{*-1}\left(\Omega^{i} X\right)$ coincides with the cohomology suspension. Lemmas 3.1 and 3.2 yield the result.

Lemma 3.4. $\alpha_{m} \eta \widetilde{\mathfrak{D}}_{m-1} \cdots \eta \widetilde{\mathfrak{D}}_{1} \eta \mathfrak{D}(\operatorname{Ker} q)=0$.

Proof. Let $\pi_{1}: F^{-1} H^{*}\left(\Lambda X ; \mathbb{F}_{p}\right) \rightarrow{ }_{1} E_{\infty}^{-1, *}$ be the natural projection. From the commutativity of the diagram (i) of Lemma 3.2, it follows that $\pi_{1} \widetilde{\alpha}_{1}(q \otimes 1) \mathfrak{D}(x)=0$ for any $x \in \operatorname{Ker} q$. Therefore we see that $\widetilde{\alpha}_{1}(q \otimes 1) \mathfrak{D}(x)+e v_{1}^{*} y=0$ for some $y \in H^{*}\left(X ; \mathbb{F}_{p}\right)$. Since $c_{1}^{*} e v_{1}^{*}=i d$, it follows from the definition of $\widetilde{\alpha}_{1}$ that $y=0$. Thus $\widetilde{\alpha}_{1}(q \otimes 1) \mathfrak{D}(x)=0$ and hence $\alpha_{1} \eta \mathfrak{D}(x)=0$. The same argument still works well on the diagram (ii) of Lemma 3.2. In consequence we have $\alpha_{i} \eta \widetilde{\mathfrak{D}}_{i-1}(z)=0$ for $z \in \operatorname{Ker} \alpha_{i-1}$. This completes the proof.

Proof of Theorem 2.2. We can choose a basis $S$ for $H^{*}\left(\Omega^{m} X ; \mathbb{F}_{p}\right)$ extending that of $\left(\sigma^{*}\right)^{(m)}(V)$, say $\left\{\left(\sigma^{*}\right)^{(m)} x_{1}, \ldots,\left(\sigma^{*}\right)^{(m)} x_{s}, ..\right\} \cup\left\{b_{1}, b_{2}, ..\right\}$, where $\left\{x_{1}, . ., x_{s}, ..\right\}$ is 
a basis for $V$ with $\operatorname{deg} x_{1} \leqslant \operatorname{deg} x_{2} \leqslant \cdots$. Suppose that the evaluation fibration $\Omega^{m} X \rightarrow \Lambda^{m} X \rightarrow X$ is TNCZ with respect to $\mathbb{F}_{p}$. Then there exists an isomorphism $\Phi: H^{*}\left(\Lambda^{m} X ; \mathbb{F}_{p}\right) \rightarrow H^{*}\left(X ; \mathbb{F}_{p}\right) \otimes H^{*}\left(\Omega^{m} X ; \mathbb{F}_{p}\right)$ of an $H^{*}\left(X ; \mathbb{F}_{p}\right)$-module such that $i n_{2}^{*} \Phi=j_{m}^{*}$, where $i n_{2}: \Omega^{m} X \rightarrow X \times \Omega^{m} X$ is the inclusion into the second factor. We here consider the image of $\left(s^{-1}\right)^{(m)} x_{i}$ by $i n_{2}^{*} \Phi \alpha_{m}$. From Lemma 3.3, it follows that $i n_{2}^{*} \Phi \alpha_{m}\left(\left(s^{-1}\right)^{(m)} x_{i}\right)=j^{*} \alpha_{m}\left(\left(s^{-1}\right)^{(m)} x_{i}\right)=\left(\sigma^{*}\right)^{(m)} x_{i}$. Therefore we can write

$$
\Phi \alpha_{m}\left(\left(s^{-1}\right)^{(m)} x_{i}\right)=\left(\sigma^{*}\right)^{(m)} x_{i}+\sum_{z \in S, \operatorname{deg} z<\operatorname{deg} x_{i}-m} Q_{(z, i)} z
$$

where $Q_{(z, i)}$ are appropriate elements of $H^{\geqslant 1}\left(X ; \mathbb{F}_{p}\right)$. Since $\eta \mathfrak{D} \rho \neq 0$ by the assumption, we see that the element $\eta \mathfrak{D}(\rho)$ is expressed as $\sum_{i=1}^{k} P_{i} s^{-1} x_{i}$ with elements $P_{i}$ of $H^{*}\left(X ; \mathbb{F}_{p}\right)$ in which $P_{k} \neq 0$. Letting $\widetilde{\eta}=\eta \widetilde{\mathfrak{D}}_{m-1} \cdots \eta \widetilde{\mathfrak{D}}_{1} \eta \mathfrak{D}$, then Lemma 2.4 implies that $\widetilde{\eta}(\rho)=\sum_{i=1}^{k} P_{i}\left(s^{-1}\right)^{(m)} x_{i}$. From Lemma 3.4, it turns out that

$$
\begin{aligned}
0=\Psi \alpha_{m} \eta(\rho) & =\sum_{i=1}^{k} P_{i}\left(\left(\sigma^{*}\right)^{(m)} x_{i}+\sum_{z \in S, \operatorname{deg}} \sum_{z<\operatorname{deg} x_{i}-m} Q_{(z, i)} z\right) \\
=P_{k}\left(\left(\sigma^{*}\right)^{(m)} x_{k}+\sum_{z \in S, \operatorname{deg} z<\operatorname{deg} x_{k}-m} Q_{(z, k)} z\right) & \\
& +\sum_{i=1}^{k-1} P_{i}\left(\left(\sigma^{*}\right)^{(m)} x_{i}+\sum_{z \in S, \operatorname{deg} z<\operatorname{deg} x_{i}-m} Q_{(z, i)} z\right) .
\end{aligned}
$$

The fact that $H^{*}\left(X ; \mathbb{F}_{p}\right) \otimes H^{*}\left(\Omega^{m} X ; \mathbb{F}_{p}\right)$ is a free $H^{*}\left(X ; \mathbb{F}_{p}\right)$-module enables us to deduce that $P_{k}=0$, which is a contradiction.

\section{Proofs of Theorems $1.1,1.2$ and 1.3}

Proof of Theorem 1.1.. Let $\left\{E^{r}, d^{r}\right\}$ and $\left\{\tilde{E}^{r}, \tilde{d}^{r}\right\}$ be the integral homology LeraySerre spectral sequence for the evaluation fibration $\mathcal{F}_{m}$ and its $\bmod p$ reduction, respectively. From [8, Theorem 3.5] and a result due to Hansen [5], we can obtain the following commutative diagram (4.1):

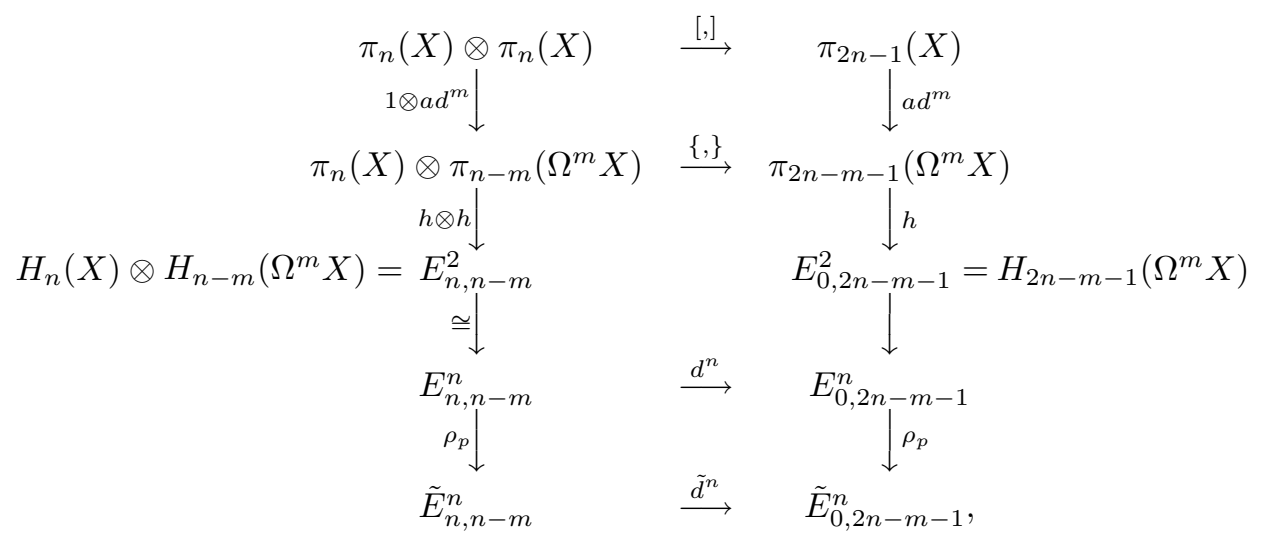


where $\{$,$\} denotes the brace product in the sense of James associated with the fibra-$ tion $\mathcal{F}_{m}$. Thus in order to prove Theorem 1.1, it suffices to show that $\operatorname{dim}\left(\operatorname{Im} \tilde{d}^{n}\right) \geqslant$ $\operatorname{dim} \operatorname{Ker} \xi$ and that $\operatorname{dim}\left(\operatorname{Im} \tilde{d}^{n}\right)=1 / 2 \cdot s(s+1)-\varepsilon s$ if $\xi^{\prime} \equiv 0$.

Let $\left\{\iota_{i}\right\}_{1 \leqslant i \leqslant s}$ be a basis for $H_{n}\left(X ; \mathbb{F}_{p}\right)$ and $\iota_{i}(m)$ the iterated transgression image of the element $\iota_{i}$ in $H_{n-m}\left(\Omega^{m} X ; \mathbb{F}_{p}\right)$. We denote by $x^{*}$ the dual element to an element $x$ of $H_{*}\left(X ; \mathbb{F}_{p}\right)$ or $H_{*}\left(\Omega^{m} X ; \mathbb{F}_{p}\right)$. Let $\xi^{\prime}: \mathbb{F}_{p}\left\{\iota_{i}^{*} \otimes \iota_{j}^{*} ; i-j \geqslant \varepsilon\right\} \rightarrow H^{2 n}\left(X ; \mathbb{F}_{p}\right)$ be the restriction of the cup product to the subspace of $H^{n}\left(X ; \mathbb{F}_{p}\right) \otimes H^{n}\left(X ; \mathbb{F}_{p}\right)$ generated by the elements $\iota_{i}^{*} \otimes \iota_{j}^{*}$. Observe that $\operatorname{dim} \operatorname{Ker} \xi^{\prime}=\operatorname{dim} \operatorname{Ker} \xi$. For a basis $\left\{b_{l}\right\}$ of Ker $\xi^{\prime}$, we write $b_{l}=\sum_{i-j \geqslant \varepsilon} \alpha_{l}^{i j} \iota_{i}^{*} \otimes \iota_{j}^{*}$. Since $\sum \alpha_{l}^{i j} \iota_{i}^{*} \cdot \iota_{j}^{*}=\xi^{\prime}\left(b_{l}\right)=0$, it follows from Lemmas 2.4 and 3.4 that (4.2):

$$
\begin{aligned}
0 & =\alpha_{m} \eta \widetilde{\mathfrak{D}}_{m-1} \cdots \eta \widetilde{\mathfrak{D}}_{1} \eta \mathfrak{D}\left(\xi^{\prime}\left(b_{l}\right)\right) \\
& =(-1)^{n(m-1)} \sum \alpha_{l}^{i j}\left\{\iota_{j}^{*} \cdot \overline{\iota_{i}(m)^{*}}+(-i)^{n} \iota_{i}^{*} \cdot \overline{\iota_{j}(m)^{*}}\right\},
\end{aligned}
$$

where $\overline{\iota_{i}(m)^{*}}$ is the element of $H^{*}\left(\Lambda^{m} X ; \mathbb{F}_{p}\right)$ such that $j_{m}^{*}\left(\overline{\iota_{i}(m)^{*}}\right)=\iota_{i}(m)^{*}$. We look at the mod $p$ cohomology Leray-Serre spectral sequence $\left\{E_{r}, d_{r}\right\}$ for $\mathcal{F}_{m}$. Let $V$ be the subspace of $E_{2}^{n, n-m}$ generated by the elements $\sum \alpha_{l}^{i j}\left\{\iota_{j}^{*} \otimes \iota_{i}(m)^{*}+(-i)^{n} \iota_{i}^{*} \otimes\right.$ $\left.\iota_{j}(m)^{*}\right\}$. Since $e v^{*}$ is a monomorphism, every element of $V$ is a permanent cycle. The equality (4.2) implies that all elements in $V$ are in the image by the differential $d_{n}: E_{n}^{0,2 n-m-1} \rightarrow E_{n}^{n, n-m}$. Therefore, letting $\operatorname{dim} H^{2 n-m-1}\left(\Omega^{m} X ; \mathbb{F}_{p}\right)=k$ and $\operatorname{dim} H^{2 n-m-1}\left(X ; \mathbb{F}_{p}\right)=l$, we have $\operatorname{dim} H^{2 n-m-1}\left(\Lambda^{m} X ; \mathbb{F}_{p}\right) \leqslant k-\operatorname{dim} \operatorname{Ker} \xi^{\prime}+$ $l$ because $\operatorname{dim} V=\operatorname{dim} \operatorname{Ker} \xi^{\prime}$. We again turn to the $\bmod p$ homology spectral sequence $\left\{\tilde{E}^{r}, \tilde{d}^{r}\right\}$. The map $e v_{*}$ is an epimorphism so that every element in $\tilde{E}_{*, 0}^{2}=$ $H_{*}\left(X ; \mathbb{F}_{p}\right)$ is a permanent cycle. Thus we have $\operatorname{dim} H_{2 n-m-1}\left(\Lambda^{m} X ; \mathbb{F}_{p}\right)=k-$ $\operatorname{dim} \operatorname{Im} \tilde{d}_{n, n-m}^{n}+l$ and hence (4.3): $\operatorname{dim} \operatorname{Im} \tilde{d}_{n, n-m}^{n} \geqslant \operatorname{dim} \operatorname{Ker} \xi^{\prime}$. We have the former half of Theorem 1.1. Suppose that $\xi \equiv 0$. Then $\operatorname{dim} \operatorname{Ker} \xi^{\prime}=1 / 2 \cdot s(s+1)-\varepsilon s$. Since the Whitehead product is skew commutative, it follows from the diagram (4.1) that $\tilde{d}^{n}\left(\iota_{i} \otimes \iota_{j}(m)\right)=\tilde{d}^{n}\left(\iota_{j} \otimes \iota_{i}(m)\right)$, further $\tilde{d}^{n}\left(\iota_{i} \otimes \iota_{j}(m)\right)=\tilde{d}^{n}\left(\iota_{j} \otimes \iota_{i}(m)\right)=0$ if $n$ is odd. Thus we see that $\operatorname{dim} \operatorname{Im} \tilde{d}_{n, n-m}^{n} \leqslant 1 / 2 \cdot s(s+1)-\varepsilon s$. Combining this inequality with (4.3), we can conclude that $\operatorname{dim} \operatorname{Im} \tilde{d}_{n, n-m}^{n}=1 / 2 \cdot s(s+1)-\varepsilon$.

If $X$ is $(n-1)$-connected, then the Hurewicz homomorphism $h: \pi_{n+1}(X) \rightarrow$ $H_{n+1}(X)$ is surjective. Therefore, in the homology Leray-Serre spectral sequence $\left\{E^{r}, d^{r}\right\}$ in the above proof, every element of $E_{n, n+1-m}^{2}$ and $E_{n+1, n-m}^{2}$ is spherical, that is; the element is in the image of the the Hurewicz homomorphism. With this in mind, we shall prove Theorem 1.2. The proof is similar to that of Theorem 1.1 although a little complicated argument is needed. In fact, we have to consider two differentials $\tilde{d}^{n+1}: \tilde{E}_{n+1, n-m}^{n+1} \rightarrow \tilde{E}_{0,2 n-m}^{n+1}$ and $\tilde{d}^{n}: \tilde{E}_{n, n+1-m}^{n} \rightarrow \tilde{E}_{0,2 n-m}^{n}$ in the mod $p$ homology Leray-Serre spectral sequence $\left\{\tilde{E}^{r}, \tilde{d}^{r}\right\}$.

Proof of Theorem 1.2.. Under the notation in the proof of Theorem 1.1, let us consider the commutative diagram (4.2): 


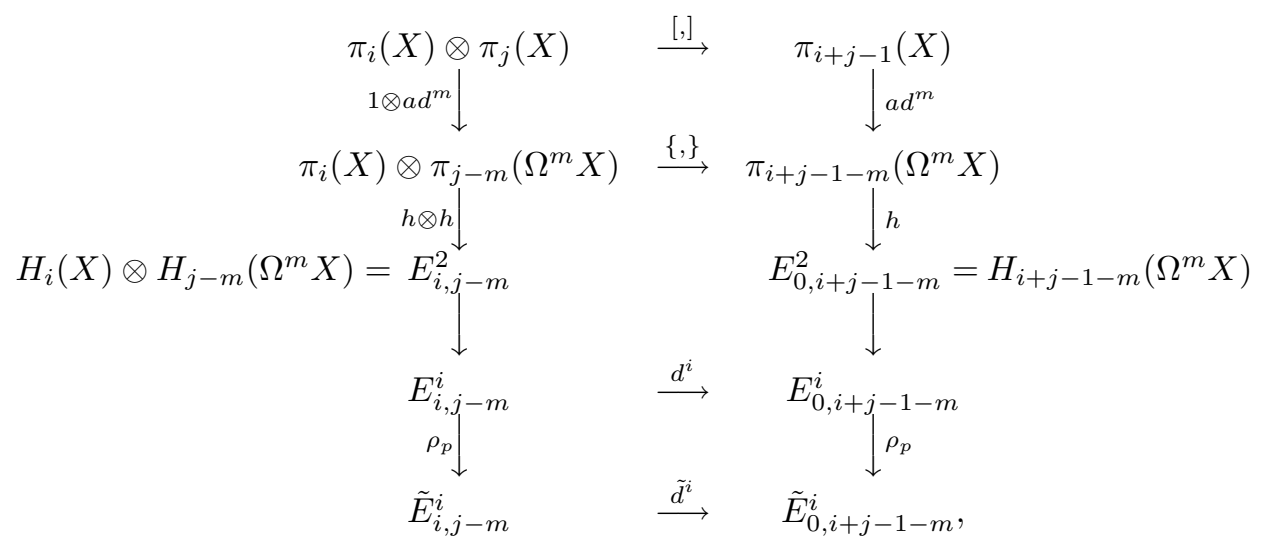

for $i=n$ and $j=n+1$. Observe that the composition of left vertical arrows is surjective. As in the proof of Theorem 1.1, it suffices to show that $\operatorname{dim}\left(\operatorname{Im} \tilde{d}^{n}\right) \geqslant$ Ker $\zeta$. By comparing the dimension of the $E^{\infty}$-term of $\left\{\tilde{E}^{r}, \tilde{d}^{r}\right\}$ with that of the $E_{\infty}$-term of $\left\{E_{r}, d_{r}\right\}$ on the total degree $2 n-1-m$, we see that

$$
\operatorname{dim} \operatorname{Im}\left\{\tilde{d}^{n}: \tilde{E}_{n, n-m}^{n} \rightarrow \tilde{E}_{0,2 n-m-1}^{n}\right\}=\operatorname{dim} \operatorname{Im}\left\{d_{n}: E_{n}^{0,2 n-m-1} \rightarrow E_{n}^{n, n-m}\right\} .
$$

Put $k=\operatorname{dim} H^{2 n-m}\left(\Omega^{m} X ; \mathbb{F}_{p}\right)$ and $l=\operatorname{dim} H^{2 n-m}\left(X ; \mathbb{F}_{p}\right)$. Then it follows that

$$
\begin{array}{r}
\operatorname{dim} H^{2 n-m}\left(\Lambda^{m} X ; \mathbb{F}_{p}\right)=k-\operatorname{dim} d_{n}^{0,2 n-m}-\operatorname{dim} d_{n+1}^{0,2 n-m} \\
+\operatorname{dim} \operatorname{coker} d_{n}^{0,2 n-m-1}+l \\
\leqslant k-\operatorname{dim} d_{n}^{0,2 n-m}+\operatorname{dim} E_{2}^{n, n-m} \\
-\operatorname{dim} \operatorname{Im} d_{n}^{0,2 n-m-1}+l .
\end{array}
$$

On the other hand, $\operatorname{dim} H_{2 n-m}\left(\Lambda^{m} X ; \mathbb{F}_{p}\right)=k-\operatorname{dim} \tilde{d}_{n, n+1-m}^{n}-\operatorname{dim} \tilde{d}_{n+1, n-m}^{n+1}+$ $\operatorname{dim} E_{n, n-m}^{2}-\operatorname{dim} \operatorname{Im} \tilde{d}_{n, n-m}^{n}+l$. We here consider the commutative diagram (4.2) for $i=n+1$ and $j=n$. Since the composition of the left vertical arrows is also surjective in this case, the skew commutativity of the Whitehead product enables us to deduce that $\operatorname{Im} \tilde{d}_{n+1, n-m}^{n+1}=0$. It turns out that $\operatorname{dim} \operatorname{Im} d_{n}^{0,2 n-m} \leqslant$ $\operatorname{dim} \operatorname{Im} \tilde{d}_{n, n+1-m}^{n}$. We are left to show that $\operatorname{dim} \operatorname{Ker} \zeta \leqslant \operatorname{dim} \operatorname{Im} d_{n}^{0,2 n-m}$. Since the Hurewicz map $h: \pi_{i}(X) \rightarrow H_{i}(X)$ is an isomorphism for $i=n$ and an epimorphism for $i=n+1$, it follows that the iterated cohomology suspension $\left(\sigma^{*}\right)^{(m)}: H^{i}\left(X ; \mathbb{F}_{p}\right) \rightarrow H^{i-m}\left(\Omega^{m} X ; \mathbb{F}_{p}\right)$ is a isomorphism for $i=n$ and a monomorphism for $i=n+1$. Lemma 3.3 implies that every element $x$ in the image of the map $\left(\sigma^{*}\right)^{(m)}: H^{n+1}\left(X ; \mathbb{F}_{p}\right) \rightarrow H^{n+1-m}\left(\Omega^{m} X ; \mathbb{F}_{p}\right)$ is also in $\operatorname{Im} j_{m}^{*}$. Therefore the element $x$ is a permanent cycle. As in the proof of Theorem 1.1, applying the map $\alpha_{m} \eta \widetilde{\mathfrak{D}}_{m-1} \cdots \eta \widetilde{\mathfrak{D}}_{1} \eta \mathfrak{D}$ to appropriate elements of $\operatorname{Ker} \zeta$, we have non-trivial relations between indecomposable elements of $H^{*}\left(\Lambda^{m} X ; \mathbb{F}_{p}\right)$. In the cohomology Leray-Serre spectral sequence, the relations are caused by the differential $d_{n}^{0,2 n-m}: E_{n}^{0,2 n-m} \rightarrow E_{n}^{n, n+1-m}$. Thus the same argument as in the proof of Theorem 1.1 enables us to obtain the required inequality. 
Remark 4.1. The proof of [2, Theorem 2] relies on the bijectivity of the Hurewicz homomorphism [2, page 259, line 6]. So it does not seem to be easy to improve the proof due to Chen for showing Theorem 1.2.

The rest of this section is devoted to proving Theorem 1.3. We first recall an equality related to the differential of the minimal model for $X$ and the Whitehead product. Let $(\wedge V, d)$ be the minimal model for the given $(n-1)$-connected space $X$. It is known that there exists a non-degenerate $\mathbb{Q}$-bilinear map $\langle;\rangle: V^{s} \times \pi_{s}(X) \otimes_{\mathbb{Z}}$ $\mathbb{Q} \rightarrow \mathbb{Q}$ for any $s \geqslant 2$. We define another bilinear map $\langle;\rangle_{2}: \wedge^{2} V \times\left(\pi_{*}(X) \otimes_{\mathbb{Z}} \mathbb{Q}\right) \otimes$ $\left(\pi_{*}(X) \otimes_{\mathbb{Z}} \mathbb{Q}\right) \rightarrow \mathbb{Q}$ by $\left\langle v \wedge w ; \gamma_{0} \otimes \gamma_{1}\right\rangle_{2}=\left\langle v ; \gamma_{1}\right\rangle\left\langle w ; \gamma_{0}\right\rangle+(-1)^{\operatorname{deg} w \operatorname{deg} \gamma_{0}}\left\langle v ; \gamma_{1}\right\rangle\left\langle w ; \gamma_{0}\right\rangle$. The linear map $[\text {, ] }]_{\mathbb{Q}}: \pi_{*}(X) \otimes_{\mathbb{Z}} \mathbb{Q} \otimes \pi_{*}(X) \otimes_{\mathbb{Z}} \mathbb{Q} \rightarrow \pi_{*}(X) \otimes_{\mathbb{Z}} \mathbb{Q}$ is defined by $\left[\gamma_{0} \otimes q_{0}, \gamma_{1} \otimes q_{1}\right]_{\mathbb{Q}}=\left[\gamma_{0}, \gamma_{1}\right] \otimes q_{0} q_{1}$, where [, ] is the Whitehead product, $q_{i} \in \mathbb{Q}$ and $\gamma_{i} \in \pi_{*}(X)$. Observe that $(\operatorname{Im}[],) \otimes_{\mathbb{Z}} \mathbb{Q}=\operatorname{Im}[,]_{\mathbb{Q}}$. We then obtain the equality (4.3):

$$
\left\langle d_{1} v ; \gamma_{0} \otimes \gamma_{1}\right\rangle_{2}=(-1)^{k+s-1}\left\langle v ;\left[\gamma_{0}, \gamma_{1}\right]_{\mathbb{Q}}\right\rangle,
$$

where $v \in V, \gamma_{0} \in \pi_{k}(X) \otimes_{\mathbb{Z}} \mathbb{Q}, \gamma_{1} \in \pi_{s}(X) \otimes_{\mathbb{Z}} \mathbb{Q}$ and $d_{1}$ is the quadratic part of the differential $d$ (see [4, Proposition 13.16]). To simplify, the composition $V^{n} \otimes V^{n+1} \times$ $\left(\pi_{n}(X) \otimes_{\mathbb{Z}} \mathbb{Q}\right) \otimes\left(\pi_{n+1}(X) \otimes_{\mathbb{Z}} \mathbb{Q}\right) \stackrel{\cong}{\longrightarrow}\left(\wedge^{2} V\right)^{2 n+1} \times \pi_{*}(X) \otimes_{\mathbb{Z}} \mathbb{Q} \otimes \pi_{*}(X) \otimes_{\mathbb{Z}} \mathbb{Q} \stackrel{\langle;\rangle_{2}}{\longrightarrow} \mathbb{Q}$ is denoted by $\langle;\rangle^{\prime}$ below. It is immediate that $\left\langle v \otimes w ; \gamma_{0} \otimes \gamma_{1}\right\rangle^{\prime}=\left\langle v ; \gamma_{1}\right\rangle\left\langle w ; \gamma_{0}\right\rangle$ for $v \otimes w \in V^{n} \otimes V^{n+1}$. Therefore, the map $\langle;\rangle^{\prime}$ is also non-degenerate. Thus the equality (4.3) yields that $\operatorname{dim} \operatorname{Im} d_{1}=\operatorname{dim}\left(\operatorname{Im}[,] \otimes_{\mathbb{Z}} \mathbb{Q}\right)$. We note that $V^{n} \cong$ $H^{n}(X ; \mathbb{Q})$ and $V^{n+1}=\hat{V}^{n+1} \oplus \mathbb{Q}\left\{v_{\alpha}\right\}$ for which $\hat{V}^{n+1} \cong H^{n+1}(X ; \mathbb{Q}), \mathbb{Q}\left\{v_{\alpha}\right\}=0$ if $n \neq 2$ and $\mathbb{Q}\left\{v_{\alpha}\right\} \cong$ Ker $m_{2}$ if $n=2$. Moreover, we see that the map $\zeta$ : $H^{n}(X ; \mathbb{Q}) \otimes H^{n+1}(X ; \mathbb{Q})=V^{n} \otimes V^{n+1} \rightarrow H^{2 n+1}(X ; \mathbb{Q})$ factors through the injection $\bar{\zeta}: V^{n} \otimes V^{n+1} \rightarrow(\operatorname{Ker} d)^{2 n+1}$. For dimensional reasons, we can write $(\operatorname{Ker} d)^{2 n+1}=$ $\mathbb{Q}\left\{v_{i}^{n} \cdot v_{j}^{n+1}\right\} \oplus \mathbb{Q}\left\{w_{\beta}\right\} \oplus \mathbb{Q}\left\{\sum a_{l}^{i \alpha} v_{i}^{n} v_{\alpha}\right\}$. Here $\left\{v_{i}^{n}\right\}$ and $\left\{v_{j}^{n+1}\right\}$ are bases for $V^{n}$ and $V^{n+1}$ respectively and $w_{\beta}$ are appropriate indecomposable elements. Observe that $d^{2 n}=d_{1}^{2 n}$. In general, $\bar{\zeta}(\operatorname{Ker} \zeta) \subset \operatorname{Im} d$ so that $\operatorname{dim}\left(\operatorname{Im}[,] \otimes_{\mathbb{Z}} \mathbb{Q}\right)^{2 n} \geqslant \operatorname{Ker} \zeta$. Thus we have the following proposition.

Proposition 4.2. $\operatorname{dim}\left(\operatorname{Im}[,] \otimes_{\mathbb{Z}} \mathbb{Q}\right)^{2 n}=\operatorname{Ker} \zeta$ if and only if $\bar{\zeta}(\operatorname{Ker} \zeta)=\operatorname{Im} d$.

Proof of Theorem 1.3.. We first prove (2). From the injectivity of the map $m_{2}$, we see that $\bar{\zeta}(\operatorname{Ker} \zeta)=\operatorname{Im} d$. Moreover, since $\operatorname{dim}\left(\operatorname{Im} \rho_{0} \circ h \circ a d^{m} \circ[],\right)=\operatorname{dim}(h \otimes 1 \circ$ $\left.a d^{m} \otimes 1\left(\operatorname{Im}[,] \otimes_{\mathbb{Z}} \mathbb{Q}\right)\right)$, it follows from Proposition 4.2 that $\operatorname{dim}\left(\operatorname{Im} \rho_{0} \circ h \circ a d^{m} \circ[],\right) \leqslant$ $\operatorname{dim}\left(\operatorname{Im}[,] \otimes_{\mathbb{Z}} \mathbb{Q}\right)=\operatorname{dim} \operatorname{Ker} \zeta$. The result follows from Theorem 1.2. The same argument as above, in which $V^{n+1}$ is replaced with $V^{n}$, enables us to deduce that the inequality in Theorem 1.1 becomes an equality if $p=0$.

As mentioned above, the inequality $\operatorname{dim}\left(\operatorname{Im}[,] \otimes_{\mathbb{Z}} \mathbb{Q}\right)^{2 n} \geqslant \operatorname{Ker} \zeta$ holds in general for $(n-1)$-connected space $(n \geqslant 2)$. We conclude this section with an example which asserts that the inequality can be strict.

Example 4.3. We define a minimal differential graded algebra $(\wedge V, d)$ over $\mathbb{Q}$ as follows: let $V$ be the vector space generated by the elements $x_{\alpha}, x_{\beta}, x_{\gamma}$ with degree $2, v, w_{\alpha \beta}, w_{\gamma \alpha}$ with degree 3 and $z$ with degree 4 . Define the differential $d$ by 
$d\left(x_{i}\right)=0=d(v), d\left(w_{\alpha \beta}\right)=x_{\alpha} x_{\beta}, d\left(w_{\gamma \alpha}\right)=x_{\gamma} x_{\alpha}$ and $d(z)=x_{\gamma} w_{\alpha \beta}-w_{\gamma \alpha} x_{\beta}$. Then it follows that $\operatorname{dim} \operatorname{Ker} \zeta=0$ and $\operatorname{dim}(\operatorname{Im} d)^{5}=1$. From Proposition 4.2, we can conclude that $\operatorname{dim}\left(\operatorname{Im}[,] \otimes_{\mathbb{Z}} \mathbb{Q}\right)^{2 n}>\operatorname{Ker} \zeta$.

\section{References}

[1] D. Anick, Connections between Yoneda and Pontrjagin algebras, Lecture Note in Math. vol. 1050, Springer, Berlin, 1984, pp. 331-350.

[2] K. T. Chen, On the Whitehead products, Proc. Amer. Math. Soc. 34(1972), 257-259.

[3] S. Eilenberg and J. C. Moore, Homology and fibrations I, Coalgebras, cotensor and its derived functors, Comment. math. helvet. 40(1966), 199-236.

[4] Y. Félix, S Halperin and J. -C. Thomas, Rational Homotopy Theory, Graduate Texts in Mathematics 205, Springer-Verlag.

[5] V. L. Hansen, Decomposability of evaluation fibrations and the brace product operation of James, Compo. Math. 35(1977), 83-89.

[6] I. M. James, On the decomposability of fibre spaces, LNM 168(1970), 125134.

[7] J. D. S. Jones, Cyclic homology and equivariant homology, Invent. Math. 87(1987), 403-423.

[8] S.Kallel and D.Sjerve, On brace products and the structure of fibrations with section, Proc. London Math. Soc.(3) 83(2001) 419-442.

[9] K. Kuribayashi, On the mod $p$ cohomology of spaces of free loops on the Grassmann and Stiefel manifolds, J. Math. Soc. Japan 43(1991), 331-346.

[10] K. Kuribayashi, Module derivations and the adjoint action of a finite loop space, J. Math. Kyoto Univ. 39 (1999), 67-85.

[11] M. Mimura and H. Toda, Topology of Lie groups I, Translations of Math. Monographs 91 Amer. Math. Soc. 1991.

[12] H. Samelson, On the relationship between the Whitehead product and Pontryagin product, Amer. J. Math. 75(1953), 744-752.

[13] L. Smith, Homological algebra and the Eilenberg-Moore spectral sequence, Trans. Amer. Math. Soc. 129(1967), 58-93.

[14] L. Smith, On the Künneth theorem I, Math. Z. 166(1970), 94-140. 
[15] L. Smith, On the characteristic zero cohomology of the free loop space, Amer. J. Math. 103(1981), 887-910.

[16] J. Tate, Homology of Netherian rings and local rings, Illinois J. Math. 1(1956),14-27.

[17] G. W. Whitehead, Elements of Homotopy Theory, Graduate Texts in Mathematics 61, Springer-Verlag.

This article may be accessed via WWW at http://www.rmi.acnet.ge/hha/ or by anonymous ftp at

ftp://ftp.rmi.acnet.ge/pub/hha/volumes/2002/n1a7/v4n1a7.(dvi,ps,pdf)

Katsuhiko Kuribayashi kuri@geom.xmath.ous.ac.jp

Department of Applied Mathematics, Okayama University of Science, Okayama 700-0005, Japan 